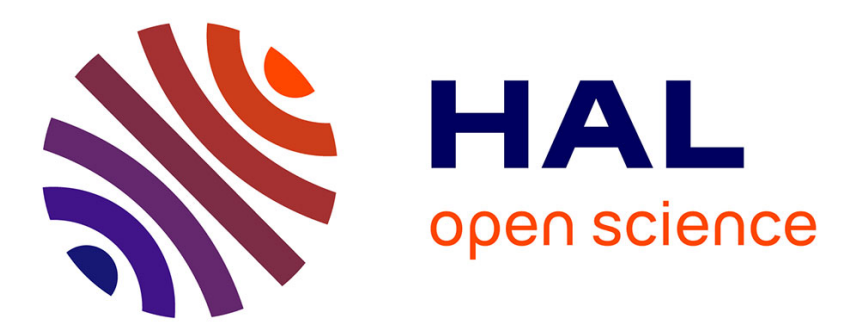

\title{
Spectroscopie d'absorption multiphotonique sans effet Doppler
}

\author{
B. Cagnac, G. Grynberg, F. Biraben
}

\section{To cite this version:}

B. Cagnac, G. Grynberg, F. Biraben. Spectroscopie d'absorption multiphotonique sans effet Doppler. Journal de Physique, 1973, 34 (10), pp.845-858. 10.1051/jphys:019730034010084500 . jpa-00207447

\section{HAL Id: jpa-00207447 https://hal.science/jpa-00207447}

Submitted on 1 Jan 1973

HAL is a multi-disciplinary open access archive for the deposit and dissemination of scientific research documents, whether they are published or not. The documents may come from teaching and research institutions in France or abroad, or from public or private research centers.
L'archive ouverte pluridisciplinaire HAL, est destinée au dépôt et à la diffusion de documents scientifiques de niveau recherche, publiés ou non, émanant des établissements d'enseignement et de recherche français ou étrangers, des laboratoires publics ou privés. 


\title{
Classification \\ Physics Abstracts \\ 5.200 \\ SPECTROSCOPIE D'ABSORPTION MULTIPHOTONIQUE SANS EFFET DOPPLER
}

\author{
B. CAGNAC, G. GRYNBERG et F. BIRABEN \\ Laboratoire de Spectroscopie Hertzienne de l'ENS, associé au CNRS, \\ Tour 12, 11, quai Saint-Bernard, Paris, France
}

(Reçu le 30 mars 1973, révisé le 16 mai)

\begin{abstract}
Résumé. - Lorsqu'un atome est porté dans un état excité par absorption de plusieurs photons, il est possible d'éliminer l'effet Doppler en choisissant une géométrie particulière pour les faisceaux Laser. Dans le cas des transitions à deux photons, on étudie la forme de la raie : on montre que les expériences sont possibles avec des puissances de Laser relativement faibles de l'ordre du watt et que les déplacements de fréquence causés par l'irradiation lumineuse restent négligeables. L'application essentielle de ces processus semble devoir être la spectroscopie fine de niveaux très excités d'un atome ainsi que l'étude de certains états métastables.
\end{abstract}

\begin{abstract}
When an atom passes from the ground state to an excited state by absorbing simultaneously several photons, it is possible to eliminate the Doppler effect by a peculiar choice of the geometry of the Laser beams. In the case of two photon transitions, we study the line-shape : we show that experiments could be done with a relatively weak power of the Laser (in the region of a few watts) and that the light-shifts are negligeable. The essential application of this effect seems to be an accurate method of Spectroscopy of very excited levels as well as some metastable levels.
\end{abstract}

Les transitions multiphotoniques, au cours desquelles un même atome absorbe simultanément plusieurs photons, ont été observées expérimentalement d'abord dans le domaine des radiofréquences [1]; il s'agissait de transitions entre sous-niveaux Zeeman dans des expériences de résonance magnétique utilisant d'intenses champs de radiofréquence. Dans le domaine de l'optique, l'observation des transitions multiphotoniques a été une conséquence du développement des Laser; la puissance et la finesse spectrale de la source lumineuse sont en effet des facteurs déterminants pour la probabilité de transition. Les premiers Laser n'étaient accordables que sur des intervalles de fréquence relativement étroits; et pour cette raison, la plupart des expériences ont porté sur des transitions à plusieurs quanta aboutissant à un continuum d'états (ionisation d'un gaz monoatomique ; transitions entre bandes d'un solide ou d'une grosse molécule) [2]. Les premières expériences sur les transitions optiques à plusieurs quanta entre niveaux liés d'un atome ont été réalisées en déplaçant légèrement la longueur d'onde d'un laser à solides [3] ; mais la récente fabrication des Laser à colorants, accordables en fréquence, permet un plus grand développement de ce type d'études [4]. Nous montrons plus loin qu'un des intérêts majeurs de ces transitions multiphotoniques dans le domaine optique est la possi- bilité d'éliminer l'élargissement Doppler en choisissant une géométrie particulière pour les faisceaux lumineux interagissant avec l'atome. Ces transitions permettront donc d'étudier les niveaux atomiques à la précision de la largeur naturelle. Un calcul rapide montre que les probabilités de transition restent importantes avec les intensités lumineuses fournies par les Laser à gaz ou à colorant en fonctionnement monomode : l'effet proposé est donc parfaitement observable. Cette méthode de spectroscopie sans effet Doppler, dont la théorie a été ébauchée par Vasilenko, Chebotaiev et Shishaev [5] dans le cas des transitions à deux photons, présente un certain nombre de possibilités nouvelles par rapport aux autres méthodes de spectroscopie sans effet Doppler : absorption saturée [6] ; résonances à trois niveaux de Feld et Javan [7] ; déflection d'un jet atomique [8]. Nous discuterons de ses possibilités d'application.

1. Principe de la méthode. - 1.1 Principe DES TRANSITIONS DIPOLAIRES ÉLECTRIQUES A DEUX QUANTA. - Un court rappel nous donnera l'occasion de préciser nos notations. Nous nous limitons pour l'instant au cas de trois niveaux d'énergie d'un atome (Fig. 1) : le niveau fondamental désigné par $\mathrm{g}$ d'énergie $\hbar \omega_{\mathrm{g}}$; un niveau excité désigné par e, d'énergie $\hbar \omega_{\mathrm{e}}$; un autre niveau excité intermédiaire désigné par $r$, 


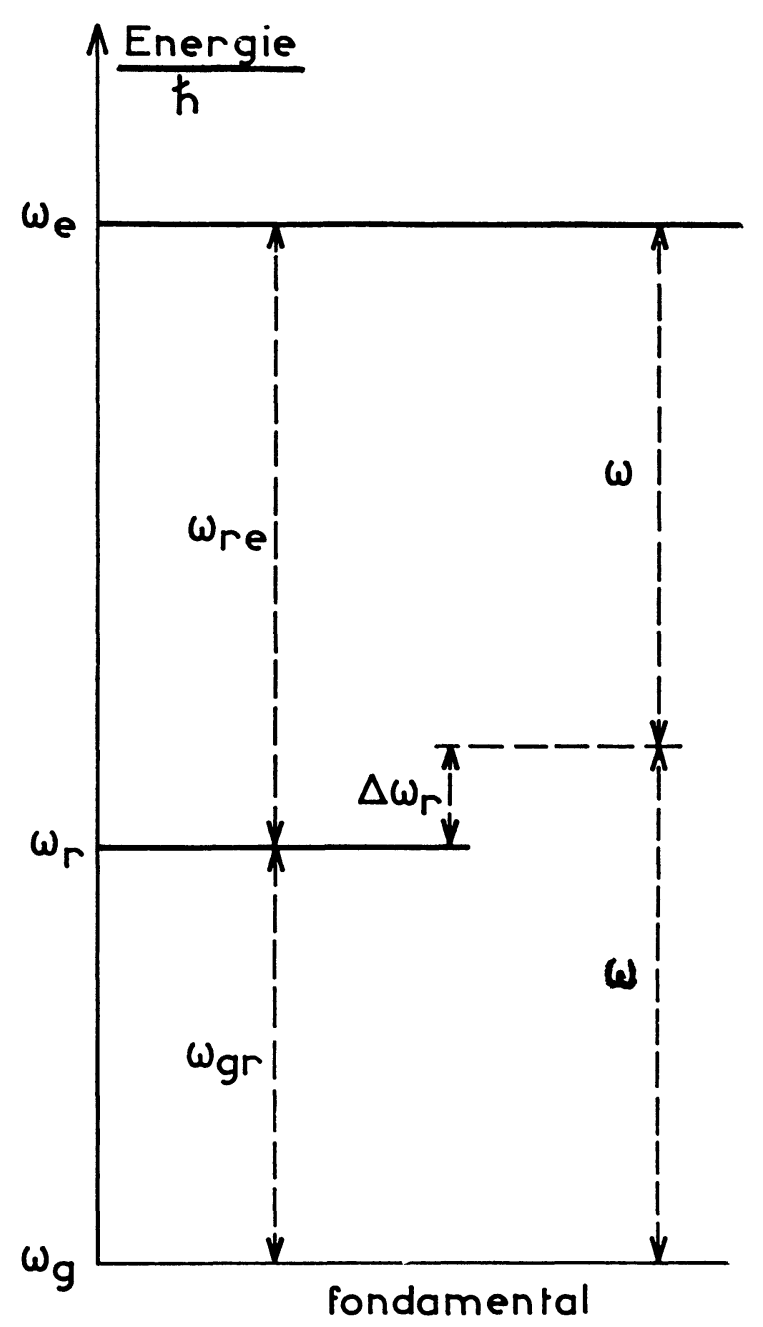

FIG. 1.

parce qu'il sera utilisé comme relais, d'énergie $\hbar \omega_{\mathrm{r}}$. Nous supposons permises les deux transitions radiatives suivantes :

1. Entre les niveaux $\mathrm{g}$ et $\mathrm{r}$ avec échange de photons d'énergie

$$
\hbar \omega_{\mathrm{gr}}=\hbar \omega_{\mathrm{r}}-\hbar \omega_{\mathrm{g}} .
$$

2. Entre les niveaux $r$ et e avec échange de photons d'énergie

$$
\hbar \omega_{\mathrm{re}}=\hbar \omega_{\mathrm{e}}-\hbar \omega_{\mathrm{r}}
$$

Nous nous limitons aux transitions dipolaires électriques : le niveau $r$ est de parité opposée aux deux niveaux e et $\mathrm{g}$. La transition directe est donc interdite entre les deux niveaux e et $\mathrm{g}$ de même parité.

Si l'on irradie l'atome supposé immobile avec une onde électromagnétique dont la pulsation $\omega$ obéit à la condition de résonance (cf. Fig. 1) :

$$
\hbar \omega_{\mathrm{ge}}=\hbar \omega_{\mathrm{e}}-\hbar \omega_{\mathrm{g}}=2 \hbar \omega .
$$

Un calcul de perturbation au second ordre [2] met en évidence la possibilité de transitions de $\mathrm{g}$ à $\mathrm{e}$, qui conservent l'énergie, avec absorption de deux photons. On peut interpréter ce processus de la manière sui- vante : en dépit du défaut d'énergie $\hbar \Delta \omega_{\mathrm{r}}=\hbar \omega-\hbar \omega_{\mathrm{gr}}$, l'atome peut passer du niveau fondamental au niveau relais en absorbant un photon; mais le temps de séjour $\Delta t$ dans ce niveau relais est très court afin de respecter le principe d'incertitude : $\Delta t . \Delta \omega_{\mathrm{r}}<1$. Si l'intensité de l'onde est assez forte pour que l'atome absorbe un second photon pendant ce temps $\Delta t$, l'atome se retrouvera dans l'état e ; et à l'issue du processus l'énergie totale aura été conservée. Il faut donc que l'intensité du faisceau lumineux soit d'autant plus importante que l'écart $\Delta \omega_{\mathrm{r}}$ est luimême plus important.

1.2 Suppression DE L'EFFET Doppler. - Considérons un atome interagissant avec deux ondes progressives de même direction, mais se propageant en sens contraire. On réalisera expérimentalement une telle situation soit en renvoyant le faisceau lumineux sur lui-même à l'aide d'un miroir, soit en plaçant la cellule d'expérience à l'intérieur de la cavité Pérot-Fabry du Laser (cf. Fig. 2a).

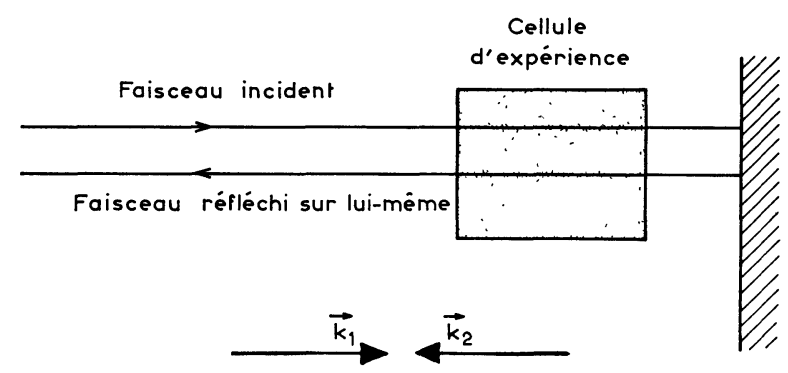

(a)

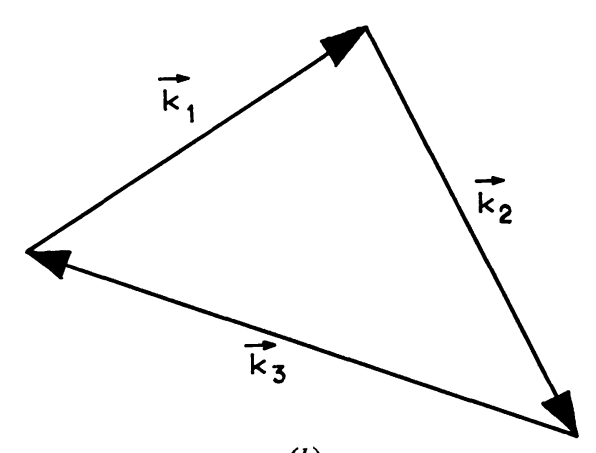

(b)

FIG. 2.

L'atome possède par rapport au laboratoire une certaine vitesse $\mathbf{v}$, dont nous appelons $v_{x}$ la compoposante sur la direction de propagation commune aux deux ondes. Dans son repère propre, l'atome est immobile, mais à cause de l'effet Doppler, il «voit » deux ondes de pulsations respectives $\omega\left(\frac{1-v_{x}}{c}\right)$ et $\omega\left(\frac{1+v_{x}}{c}\right)$; s'il absorbe un photon de chacune d'entre elles, la condition de résonance (1) s'écrit dans le repère propre sous la forme :

$\hbar \omega_{\mathrm{e}}-\hbar \omega_{\mathrm{g}}=\hbar \omega\left(1-\frac{v_{x}}{c}\right)+\hbar \omega\left(1+\frac{v_{x}}{c}\right)=2 \hbar \omega$ 
Cette condition est vérifiée simultanément par tous les atomes quelle que soit la composante $v_{x}$ de leur vitesse. Ils effectueront donc la transition à deux quanta pour la même valeur de la pulsation $\omega$. (Dans la mesure où nous avons pris le droit de négliger l'effet Doppler du second ordre en $\left(v^{2} / c^{2}\right)$; cf. Appendice I.)

Si la polarisation de l'onde aller est identique à celle de l'onde retour, les règles de sélection sont les mêmes pour l'absorption de deux photons se propageant en sens inverses ou de deux photons se propageant dans le même sens; et les deux processus sont autorisés simultanément. Mais pour l'absorption de deux photons de même sens de propagation, la condition de résonance dépend encore de la vitesse $v_{x}$. On s'attend donc à ce que la courbe représentant l'absorption en fonction de la pulsation $\omega$ ait la forme indiquée sur la figure $3 a$ : superposition d'une courbe large de faible amplitude (ayant la largeur Doppler) et d'une courbe étroite de grande amplitude (ayant la largeur naturelle).

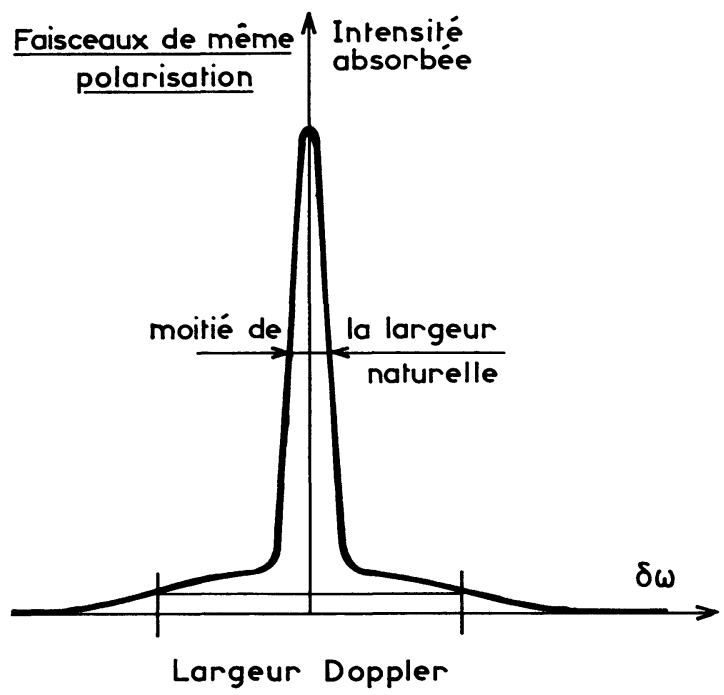

(a)

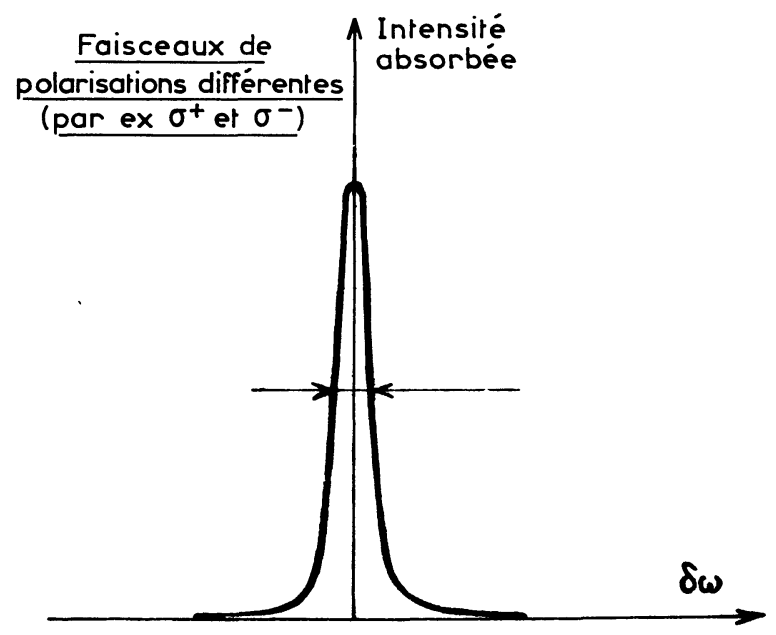

(b)

FIG. 3.
Cette suppression d'effet Doppler peut encore être comprise en terme de conservation de l'impulsion dans la collision atome-photons ; et de cette manière, il est plus facile de généraliser à un nombre quelconque de photons. Nous supposons donc l'atome soumis à plusieurs ondes progressives numérotées par l'indice $i$, et caractérisées par leurs vecteurs d'onde $\mathbf{k}_{i}$. Nous supposons en outre que la disposition géométrique des faisceaux lumineux (Fig. 2b) rend nulle l'impulsion globale d'un ensemble de photons absorbés simultanément par le même atome :

$$
\sum_{i} \hbar \mathbf{k}_{i}=0
$$

Dans ces conditions, l'impulsion de l'atome ne change pas et l'énergie des photons est entièrement utilisée à modifier l'énergie interne de l'atome, quelle que soit sa vitesse :

$$
\hbar \omega_{\mathrm{ge}}=\hbar \omega_{\mathrm{e}}-\hbar \omega_{\mathrm{g}}=\sum_{i} \hbar c\left|\mathbf{k}_{i}\right|=\sum_{i} \hbar \omega_{i}
$$

Tous les atomes effectueront la transition à $n$ quanta pour les mêmes valeurs des fréquences $\omega_{i}$. (Nous négligeons encore ici les termes du second ordre en $\left(v^{2} / c^{2}\right)$ : cf. Appendice I).

La condition vectorielle (3) redonne bien, dans le cas de deux photons, la disposition que nous avions déjà indiquée : deux ondes de sens contraires, parallèles à la même direction et de même fréquence ; la fréquence de travail est donc rigoureusement imposée. Dans le cas de trois photons, il existe un grand choix de valeurs de fréquences $\omega_{i}$ permettant de réaliser simultanément la condition vectorielle (3) et la condition arithmétique (4); il suffit en effet que l'on puisse réaliser un triangle de côtés proportionnels à $\omega_{1}, \omega_{2}$ et $\omega_{3}$, c'est-à-dire que chacune des pulsations $\omega_{i}$ soit inférieure à la somme des deux autres. On voit que cette condition est extrêmement souple. Bien qu'elles nécessitent des puissances nettement plus grandes que les transitions à deux photons, les transitions à trois photons peuvent se révéler avoir un champ d'application plus vaste.

2. Etude de la raie d'absorption à deux photons. L'expression de la probabilité de transition à deux photons est connue depuis longtemps [9], [2] ; cependant à notre connaissance, seul le calcul de la référence [5] prend en considération la vitesse des atomes et donne ainsi la forme exacte de la raie d'absorption pour un ensemble d'atomes se déplaçant dans une onde stationnaire. La forme de raie obtenue dans cette publication est cependant limitée au cas où la polarisation de l'onde aller est identique à celle de l'onde retour, or nous verrons qu'il est intéressant d'utiliser des polarisations différentes, pour les deux ondes aller et retour. D'autre part, aucune indication n'est donnée sur les déplacements des niveaux d'énergie dus à l'interaction des atomes avec les faisceaux lumineux («light-shift» [10]) or ces déplacements 
seront une cause d'erreur systématique dans les mesures spectroscopiques; il importe donc de les connaître avec précision.

Nous présentons en Appendice II un calcul de ces résonances à deux photons basé sur la théorie de l'atome "habillé » [11]. Dans cette partie, nous utilisons les principaux résultats démontrés en précisant les hypothèses nécessaires.

2.1 FORME DE RAIE. - Les photons des deux ondes progressives de sens opposés ont dans le repère du laboratoire la même énergie $\hbar \omega$; et le même module $\mathrm{du}$ vecteur d'onde $k=\omega / c$. Nous distinguerons dans le calcul les deux écarts d'énergie suivants (divisés par $\hbar$ ):

$\Delta \omega_{\mathrm{r}}=\omega-\omega_{\mathrm{gr}}$ est relativement grand; il représente la différence entre l'énergie des photons incidents et l'énergie nécessaire pour monter dans un niveau relais $|\mathrm{r}\rangle$ (cf. Fig. 1).

$\delta \omega=\omega-\frac{\omega_{\mathrm{ge}}}{2}$ est relativement petit et peut s'annuler; il représente l'écart à la condition de résonance (1) ou (4).

Les résultats présentés ci-dessous sont valables dans les conditions suivantes :

1) La vitesse des atomes est assez petite pour nous autoriser à négliger les termes quadratiques de l'effet Doppler relativiste : $v \ll c$. Dans le repère où l'atome est immobile, les deux ondes progressives aller et retour ont donc les pulsations

$$
\omega_{1}=\omega-k v_{x} \text { et } \omega_{2}=\omega+k v_{x} .
$$

Nous appellerons respectivement $\mathcal{H}_{1}$ et $\mathcal{H}_{2}$ les hamiltoniens, divisés par $\hbar$, représentant l'interaction entre l'atome et ces deux ondes.

2) Nous supposons que le niveau de départ $|g\rangle$ et le niveau d'arrivée $\mid \mathrm{e}>$ sont non dégénérés. Cette hypothèse est facile à réaliser expérimentalement en appliquant un champ magnétique assez intense pour séparer les sous-niveaux Zeeman ; mais elle n'est pas essentielle : tous les calculs développés dans l'Appendice II s'étendent, de manière évidente, au cas où le hamiltonien d'interaction entre l'atome et l'onde Laser ne couple les sous-niveaux Zeeman du niveau de départ à ceux du niveau d'arrivée qu'un à un.

3) Les transitions à un photon sont impossibles : toutes les différences $\Delta \omega_{\mathrm{r}}$ sont très grandes devant les largeurs naturelles $\Gamma_{\mathrm{r}}$ et le déplacement Doppler $k v_{x}$ :

$$
\begin{aligned}
& \Delta \omega_{\mathrm{r}} \gg k v_{x} \\
& \Delta \omega_{\mathrm{r}} \gg \Gamma_{\mathrm{r}} .
\end{aligned}
$$

4) L'amplitude des hamiltoniens $\mathfrak{H}_{1}$ et $\mathfrak{H}_{2}$ est suffisamment petite pour que la population $N_{\mathrm{g}}$ du niveau fondamental évolue peu pendant la durée de vie $1 / \Gamma_{\mathrm{e}}$ du niveau d'arrivée $\mid \mathrm{e}>$ :

$$
\Gamma_{\mathrm{g}}=-\frac{1}{N_{\mathrm{g}}} \frac{\mathrm{d} N_{\mathrm{g}}}{\mathrm{d} t} \ll I_{\mathrm{e}} .
$$

5) La projection de la vitesse de l'atome le long de la direction de propagation des faisceaux est assez grande pour que l'atome en interaction avec le rayonnement, voit plusieurs nœuds et ventres de l'onde stationnaire. Compte tenu de l'hypothèse 4), énoncée ci-dessus, cette nouvelle condition sera réalisée si :

$$
k v_{x} \gtrsim \Gamma_{\mathrm{e}} .
$$

En pratique dans tous les cas intéressants, la largeur Doppler est très grande devant la largeur naturelle et l'on ne fait pas une grande erreur en oubliant la contribution des atomes de faible vitesse dans le calcul de la forme de raie. Dans ces conditions, la probabilité de transition par unité de temps du niveau $\mid g>$ vers le niveau $\mid \mathrm{e}>$ est égale à $\left({ }^{1}\right)$ :

$$
\begin{gathered}
\Gamma_{\mathrm{g}}=\left|\sum_{\mathrm{r}} \frac{\left(\mathrm{e}\left|\mathscr{H}_{1}\right| \mathrm{r}\right)\left(\mathrm{r}\left|\mathscr{H}_{2}\right| \mathrm{g}\right)+\left(\mathrm{e}\left|\mathcal{H}_{2}\right| \mathrm{r}\right)\left(\mathrm{r}\left|\mathcal{H}_{1}\right| \mathrm{g}\right)}{\Delta \omega_{\mathrm{r}}}\right|^{2} \frac{\Gamma_{\mathrm{e}}}{4 \delta \omega^{2}+\frac{\Gamma_{\mathrm{e}}^{2}}{4}}+ \\
+\left|\sum_{\mathrm{r}} \frac{\left(\mathrm{e}\left|\mathcal{H}_{1}\right| \mathrm{r}\right)\left(\mathrm{r}\left|\mathcal{H}_{1}\right| \mathrm{g}\right)}{\Delta \omega_{\mathrm{r}}}\right|^{2} \frac{\Gamma_{\mathrm{e}}}{4\left(\delta \omega-k v_{x}\right)^{2}+\frac{\Gamma_{\mathrm{e}}^{2}}{4}}+\left|\sum_{\mathrm{r}} \frac{\left(\mathrm{e}\left|\mathcal{H}_{2}\right| \mathrm{r}\right)\left(\mathrm{r}\left|\mathcal{H}_{2}\right| \mathrm{g}\right)}{\Delta \omega_{\mathrm{r}}}\right|^{2} \frac{\Gamma_{\mathrm{e}}}{4\left(\delta \omega+k v_{x}\right)^{2}+\frac{\Gamma_{\mathrm{e}}^{2}}{4}} .
\end{gathered}
$$

On peut remarquer que, compte tenu de l'hypothèse (5), chacun des trois termes de cette formule n'est important que pour des valeurs de $\delta \omega$ qui annulent pratiquement les deux autres termes.

Nous distinguerons alors deux cas distincts :
(1) Cette formule est identique à la formule (A.5) démontrée dans l'Appendice II : $\mid \mathrm{g}$ ) représente l'état où l'atome est dans le niveau $\mathrm{g}$, en présence de $n_{1}$ photons du mode 1 et de $n_{2}$ photons du mode $2: \mid \mathrm{g})$ est égal au ket $\mid \mathrm{g}, n_{1}, n_{2}>$. De la même façon, |e) est égal à $\left.\left|\mathrm{e}, n_{1}-1, n_{2}-1>,\right| \mathrm{r}\right)$ peut, selon les cas, remplacer soit $\left|\mathrm{r}, n_{1}, n_{2}-1\right\rangle$ soit $\left|\mathrm{r}, n_{1}-1, n_{2}\right\rangle$, $\mathfrak{H}_{1}$ ne modifiant pas le nombre de photons du mode 2 (et réciproquement), il ne saurait y avoir d'ambiguïtés. 
1) Dans le cas où l'onde aller et l'onde retour ont la même polarisation et la même intensité, les éléments de matrice de $\mathfrak{H}_{1}$ et de $\mathfrak{H}_{2}$ sont identiques et la formule (5) devient :

$$
\Gamma_{\mathrm{g}}=\left|\sum_{\mathrm{r}} \frac{(\mathrm{g}|\mathcal{H}| \mathrm{r})(\mathrm{r}|\mathcal{H}| \mathrm{e})}{\Delta \omega_{\mathrm{r}}}\right|^{2}\left[\frac{4 \Gamma_{\mathrm{e}}}{4 \delta \omega^{2}+\frac{\Gamma_{\mathrm{e}}^{2}}{4}}+\frac{\Gamma_{\mathrm{e}}}{4\left(\delta \omega-k v_{x}\right)^{2}+\frac{\Gamma_{\mathrm{e}}^{2}}{4}}+\frac{\Gamma_{\mathrm{e}}}{4\left(\delta \omega+k v_{x}\right)^{2}+\frac{\Gamma_{\mathrm{e}}^{2}}{4}}\right] .
$$

On retrouve la formule de la référence [5]. Le premier terme correspond à l'absorption de deux photons se propageant en sens contraire : ce terme est indépendant de la vitesse de l'atome. Les deux autres termes correspondent à l'absorption de deux photons de la même onde progressive, ils dépendent de la vitesse de l'atome. En faisant la moyenne sur la distribution de vitesse on trouvera donc la superposition d'une lorentzienne de largeur $\Gamma_{\mathrm{e}} / 2$ et d'une gaussienne ayant la largeur Doppler habituelle. Le facteur 4 figurant au numérateur du premier terme de la formule (6) montre que la probabilité de transition "sans effet Doppler » est en moyenne double de la probabilité "avec élargissement Doppler"; en conséquence, la surface de la lorentzienne est double de la surface de la gaussienne (cf. Fig. 3a). La plupart des informations originales sur le système étant liées à la lorentzienne, les mesures seront d'autant plus précises que le rapport entre la largeur Doppler et la largeur naturelle sera plus grand.

On peut donner une interprétation physique simple de ce facteur $4:$ alors que les photons d'une même onde progressive sont indiscernables, les photons des ondes opposées sont, par contre, discernables (leur énergie étant différente dans le repère de l'atome). Lorsqu'on étudie l'amplitude de probabilité pour qu'un atome absorbe deux photons se propageant en sens opposés, il faut sommer les amplitudes correspondant aux processus "photon 1 absorbé puis photon 2 » et "photon 2 absorbé puis photon 1 ». Chacune de ces amplitudes est égale à l'amplitude d'absorber deux photons d'une même onde progres- sive. Lorsqu'on passe aux probabilités, on retrouve le facteur 4.

2) Etudions maintenant ce que devient la formule (6) quand les polarisations des ondes aller et retour sont différentes. Plus précisément, nous choisissons des polarisations $\varepsilon_{1}$ et $\varepsilon_{2}$ telles que si $\left(\mathrm{g}\left|\mathfrak{H}_{1}\right| \mathrm{r}\right)$ est différent de zéro, alors $\left(\mathrm{r}\left|\mathscr{H}_{1}\right| \mathrm{e}\right)$ est toujours nul (et ceci pour tout $\mathrm{r}$ ), la même propriété étant vraie pour $\mathfrak{H}_{2}$. On aura un exemple d'une telle situation lorsque les niveaux de départ et d'arrivée auront même nombre quantique magnétique $\left(M_{\mathrm{g}}=M_{\mathrm{e}}\right)$, les polarisations des faisceaux aller et retour étant respectivement $\sigma_{+}$et $\sigma_{-}$: il est alors évident que l'atome ne peut absorber que deux photons se propageant en sens opposés; les termes dépendant de $v_{x}$ disparaissent dans la formule (6). La raie d'absorption se réduit à la courbe lorentzienne (cf. Fig. $3 b$ ). Il est donc possible en choisissant astucieusement les polarisations d'éliminer le fond gaussien.

2.2 CalCul efFectif De La probabilité DE TRANSition. - Les expériences seront réalisées avec des Laser en fonctionnement monomode, en sorte que, dans le repère du laboratoire, les photons aient tous la même pulsation $\omega$ avec une précision bien supérieure à la largeur naturelle $\Gamma_{\mathrm{e}}$ du niveau excité. Nous calculons dans ces conditions la probabilité de transition maximum $\Gamma_{\mathrm{g}}(0)$, obtenue à la résonance exacte, $\delta \omega=0$; nous ne nous intéressons qu'à la seule raie fine, c'est-à-dire que nous considérons seulement le premier terme de l'expression (5), qui devient :

$$
\Gamma_{\mathrm{g}}(0)=\frac{4}{\Gamma_{\mathrm{e}}}\left|\sum_{\mathrm{r}} \frac{\left(\mathrm{e}\left|\mathscr{H}_{1}\right| \mathrm{r}\right)\left(\mathrm{r}\left|\mathscr{H}_{2}\right| \mathrm{g}\right)+\left(\mathrm{e}\left|\mathscr{H}_{2}\right| \mathrm{r}\right)\left(\mathrm{r}\left|\mathscr{H}_{1}\right| \mathrm{g}\right)}{\Delta \omega_{\mathrm{r}}}\right|^{2}
$$

Nous utilisons le hamiltonien d'interaction $\mathscr{H}=-\mathbf{E} \cdot \mathbf{D} / \hbar$ (cf. Appendice II), exprimé à partir $\mathrm{du}$ dipôle électrique de l'atome $\mathbf{D}=\sum q \mathbf{r}$, et $\mathrm{du}$ champ électrique de l'onde $\mathbf{E}$, parallèle au vecteur unitaire $\varepsilon$ qui caractérise sa polarisation.
Nous devons préciser les nombres quantiques caractéristiques des différents niveaux atomiques : le niveau de départ $|\mathrm{g}, J, M\rangle$, le niveau d'arrivée $\mid \mathrm{e}, J^{\prime}, M^{\prime}>$ et les niveaux intermédiaires $\mid \mathrm{r}, j_{\mathrm{r}}, m>$. L'élément de matrice s'écrit par exemple :

$$
\begin{aligned}
<\mathrm{r}, n_{1}-1\left|\mathrm{H}_{1}\right| \mathrm{g}, n_{1}> & \left.=\frac{1}{\hbar} \sqrt{\frac{n_{1} \hbar \omega}{2 \varepsilon_{0} V}}<\mathrm{r}\left|\varepsilon_{1} \cdot \mathrm{D}\right| \mathrm{g}\right\rangle \\
& =\frac{1}{\hbar} \sqrt{\frac{n_{1} \hbar \omega}{2 \varepsilon_{0} V}} \frac{\mathrm{r}\|\mathrm{D}\| \mathrm{g}\rangle}{\sqrt{2 J+1}}<j_{\mathrm{r}} 1 m q_{1} \mid J M>
\end{aligned}
$$


en introduisant l'élément de matrice réduit de l'opérateur vectoriel $\mathbf{D}$ et le coefficient de Clebsch-Gordan (l'indice $q_{1}$ exprime la polarisation de l'onde 1 ; il vaut respectivement $+1,-1$ ou 0 pour les polarisations $\sigma^{+}, \sigma^{-}$ou $\pi$ ).

$n_{1} / V$ représente le nombre de photons du mode 1 par unité de volume. Connaissant la section $S$ du faisceau Laser et la puissance $P_{1}$ transportée par l'onde progressive numéro 1 nous pouvons calculer la densité d'énergie par unité de volume

$$
\frac{n_{1}}{V} \hbar \omega=\frac{P_{1}}{S c} .
$$

Nous remplaçons les éléments de matrice réduits par les forces d'oscillateurs, définies en absorption (cf. par exemple [12]), et qui sont généralement données dans les tables de constantes:

$$
f_{\mathrm{gr}}=\frac{2 m \omega_{\mathrm{gr}}}{3 q \hbar} \cdot \frac{|\langle r\|D\| \mathrm{g}\rangle|^{2}}{2 J+1}
$$

( $q$ et $m$ sont la charge et la masse de l'électron).

Il est commode de rassembler plusieurs constantes fondamentales dans le rayon classique de l'électron

$$
r_{0}=\frac{q^{2}}{4 \pi \varepsilon_{0} m c^{2}}=2,8 \times 10^{-13} \mathrm{~cm} ;
$$

et nous obtenons pour le carré de l'élément de matrice les deux expressions suivantes :

$$
\begin{aligned}
\left.\left|<\mathrm{r}_{\mathrm{r}} m, n_{1}-1\right| \mathscr{H}_{1}\left|\mathrm{~g} J M, n_{1}\right\rangle\right|^{2} & =3 \pi r_{0} c^{2}\left(\frac{n_{1}}{V}\right) \frac{\omega}{\omega_{\mathrm{gr}}} f_{\mathrm{gr}}\left|\left\langle j_{\mathrm{r}} 1 m q_{1} \mid J M\right\rangle\right|^{2} \\
& \left.=\frac{3 r_{0}}{2 \hbar} \frac{P_{1}}{S} \lambda_{\mathrm{gr}} f_{\mathrm{gr}}\left|<j_{\mathrm{r}} 1 m q_{1}\right| J M\right\rangle\left.\right|^{2}
\end{aligned}
$$

en introduisant la longueur d'onde $\lambda_{\mathrm{gr}}=2 \pi c / \omega_{\mathrm{gr}}$.

Pour calculer numériquement $\Gamma_{\mathrm{g}}(0)$, nous simplifierons l'expression (7) en faisant deux hypothèses supplémentaires :

a) Les ondes aller et retour ont la même polarisation $\left(q_{1}=q_{2}\right)$. Ceci permet de ramener à un seul terme le numérateur de la formule (7), et de réduire la sommation sur les états $r$ à un seul sousniveau Zeeman pour chaque niveau $j_{\mathrm{r}}$

$$
\left(m=M+q_{1}=M^{\prime}-q_{1}\right) .
$$

b) Il existe un seul niveau $j_{\mathrm{r}}$ correspondant à un écart arithmétique $\left|\Delta \omega_{\mathrm{r}}\right|$ beaucoup plus petit que tous les autres; et à ce niveau $j_{\mathrm{r}}$ correspondent des forces d'oscillateur $f_{\mathrm{gr}}$ et $f_{\mathrm{re}}$ relativement importantes. Cette situation se rencontrera effectivement pour certaines transitions $\Delta J=J^{\prime}-J=2$. Dans les cas de couplage LS, en effet, on trouvera fréquemment un niveau de résonance $(L, S)$ qui tombe à peu près à mi-distance du fondamental et d'un niveau très excité ; les divers niveaux de structure fine $j_{\mathrm{r}}$ correspondant à cette multiplicité $(\mathrm{L}, \mathrm{S})$ correspondront à des écarts $\Delta \omega_{\mathrm{r}}$ du même ordre de grandeur ; mais un seul d'entre eux $j_{\mathrm{r}}=\left(J+J^{\prime}\right) / 2$ peut servir de niveau relais dans une transition $\Delta J=2$.

Avec ces hypothèses simplificatives la sommation sur $r$ se réduit à un seul terme et nous obtenons :

$$
\Gamma_{\mathrm{g}}(0)=\frac{36 r_{0}^{2}}{\Gamma_{\mathrm{e}} \hbar^{2}} \cdot \frac{P_{1} P_{2}}{S^{2}} \cdot \frac{\lambda_{\mathrm{gr}} \lambda_{\mathrm{re}}}{\Delta \omega_{\mathrm{r}}^{2}} f_{\mathrm{gr}} f_{\mathrm{re}}\left|<J^{\prime} 1 M^{\prime} q_{1}\right| j_{\mathrm{r}} m><j_{\mathrm{r}} 1 m q_{1}|J M>|^{2} .
$$

Nous sommes dans des conditions où $\Delta \omega_{\mathrm{r}} / \omega$ est faible devant l'unité et où il y a peu de différence entre les diverses longueurs d'onde $\lambda_{\mathrm{gr}} \simeq \lambda_{\mathrm{re}} \simeq \lambda=\frac{2 \pi c}{\omega}$; ce qui permet d'écrire en admettant encore que les faisceaux aller et retour ont la même intensité $\left(P_{1}=P_{2}=P\right)$ :

$$
\Gamma_{\mathrm{g}}(0) \simeq \frac{1}{\Gamma_{\mathrm{e}}}\left(\frac{r_{0} \lambda^{2}}{\hbar c} \frac{P}{S}\right)^{2}\left(\frac{\omega}{\Delta \omega_{\mathrm{r}}}\right)^{2} f_{\mathrm{gr}} f_{\mathrm{re}}\left|<J^{\prime} 1 M^{\prime} q_{1}\right| j_{\mathrm{r}} m><j_{\mathrm{r}} 1 m q_{1}|J M>|^{2} .
$$

Nous faisons un calcul d'ordre de grandeur en admettant que la carré du produit des coefficients de Clebsch-Gordan est de l'ordre de 1/10 et en adoptant les valeurs numériques suivantes :

$$
\begin{gathered}
\dot{\lambda} \simeq 5000 \AA ; \quad \frac{\Delta \omega_{\mathrm{r}}}{\omega} \simeq \frac{1}{10} ; \\
f_{\mathrm{gr}}=f_{\mathrm{re}}=1 \text { et } \Gamma_{\mathrm{e}}=10^{+8} \mathrm{~s}^{-1} . \\
\frac{P}{S}=1 \mathrm{~W} / \mathrm{mm}^{2} .
\end{gathered}
$$

On obtient $\Gamma_{\mathrm{g}}(0) \simeq 50 \mathrm{~s}^{-1}$. Il s'agit d'un phénomène tout à fait observable. Sans doute, notre calcul numérique est-il fait avec des forces d'oscillateur maximum; mais nous disposons d'une réserve de puissance puisque des Laser à colorant fonctionnant en impulsion fournissent une puissance d'environ $1 \mathrm{~kW}$ dans un seul mode. Il est facile d'autre part de focaliser le faisceau Laser pour diminuer sa section $S$ : le nombre d'atomes irradiés diminue comme $S$; mais la probabilité de transition augmente comme $1 / S^{2}$, de telle sorte que le nombre total de transitions produites augmente comme $1 / S$. 
Remarque. - Si nous avions utilisé les éléments de matrice du hamiltonien d'interaction sous la forme $\mathfrak{H e}=-(q / m) \mathbf{p . A} / \hbar$, nous aurions obtenu une expression un peu différente de la formule (11), et qui s'en déduit en la multipliant par le facteur suivant, voisin de l'unité :

$$
\begin{aligned}
{\left[\frac{\omega_{\mathrm{gr}} \cdot \omega_{\mathrm{re}}}{\omega^{2}}\right]^{2} } & \simeq\left[\frac{\left(\omega-\Delta \omega_{\mathrm{r}}\right)\left(\omega+\Delta \omega_{\mathrm{r}}\right)}{\omega^{2}}\right]^{2}= \\
& =\left[1-\frac{\Delta \omega_{\mathrm{r}}^{2}}{\omega^{2}}\right]^{2} \simeq 1-2 \frac{\Delta \omega_{\mathrm{r}}^{2}}{\omega^{2}} .
\end{aligned}
$$

Les deux hamiltoniens conduisent rigoureusement au même résultat lorsqu'on effectue correctement la sommation sur tous les niveaux $r$ [13]. L'écart de ce facteur par rapport à l'unité peut donner une idée de l'importance de l'erreur commise en réduisant la sommation à un seul terme.

2.3 Règles DE SÉLECTION. - L'intensité de la raie d'absorption à deux photons est proportionnelle à la quantité (cf. Appendice II, formule A.11).

$$
I=\left|\sum_{\mathrm{r}} \frac{\left.\left\langle\mathrm{e}\left|\varepsilon_{1} \cdot \mathrm{D}\right| \mathrm{r}\right\rangle\left\langle\mathrm{r}\left|\varepsilon_{2} . \mathrm{D}\right| \mathrm{g}\right\rangle+<\mathrm{e}\left|\varepsilon_{2} . \mathrm{D}\right| \mathrm{r}\right\rangle\left\langle\mathrm{r}\left|\varepsilon_{1} . \mathrm{D}\right| \mathrm{g}\right\rangle}{\omega-\omega_{\mathrm{r}}}\right|^{2} .
$$

(Pour simplifier, nous avons choisi $\omega_{\mathrm{g}}=0$.)

Cette quantité $I$ peut se mettre sous une forme beaucoup plus condensée en introduisant l'opérateur $Q_{12}$ défini par:

$$
\begin{gathered}
Q_{12}=\varepsilon_{1} \cdot \mathbf{D} \frac{1}{\omega-\mathscr{H}_{0}} \varepsilon_{2} \cdot \mathbf{D}+\varepsilon_{2} \cdot \mathbf{D} \frac{1}{\omega-\mathscr{H}_{0}} \varepsilon_{1} \cdot \mathbf{D} \\
I=|<\mathrm{e}| Q_{12}|\mathrm{~g}>|^{2}
\end{gathered}
$$

$\mathscr{H}_{0}$ est le hamiltonien de l'atome seul, en l'absence de rayonnement, divisé par $\hbar$; D est un opérateur vectoriel, et $1 /\left(\omega-\mathfrak{H}_{0}\right)$ est un opérateur scalaire $\left(^{2}\right)$; donc les $Q_{12}$ forment un ensemble d'opérateurs tensoriels de rang 2. De plus, les $Q_{12}$ sont des opérateurs symétriques, ils se décomposent donc uniquement sur des opérateurs tensoriels irréductibles de rang 2 et 0 .

$$
Q=a T^{2}+b T^{0} .
$$

Nous supposons que les vecteurs polarisations $\varepsilon_{1}$ et $\varepsilon_{2}$ correspondent aux polarisations $\sigma^{+}, \sigma^{-}$ou $\pi$; dans ces conditions, $Q_{12}$ s'exprime (13) en fonction des composantes standard $D_{q_{1}}$ et $D_{q_{2}}$ de l'opérateur vectoriel $\mathbf{D}$, les indices $q_{1}$ et $q_{2}$ valant respectivement $+1,-1$ ou 0 pour les polarisations $\sigma^{+}, \sigma^{-}$ou $\pi$. Il est clair que tout opérateur $T_{q}^{k}$ construit à partir de $D_{q_{1}}$ et $D_{q_{2}}$ vérifie nécessairement :

$$
q=q_{1}+q_{2} .
$$

Supposons que l'atome est dans un état de couplage LS : étudions s'il y a des règles de sélection plus sophistiquées que la règle de sélection évidente :

(2) Dans la suite de l'article, nous supposons implicitement que $\left|\omega-\omega_{\mathrm{r}}\right|$ est très grand devant la structure fine du niveau $\mathrm{r}$ : il s'ensuit que le commutateur $\left[1 /\left(\omega-\mathfrak{H}_{0}\right), \mathrm{L}\right]$ est pratiquement nul. Si $\left|\omega-\omega_{\mathbf{r}}\right|$ est de l'ordre de grandeur de la structure fine du niveau $r$, on n'a plus le droit de considérer indépendamment les variables orbitales et les variables de spin : $1 /\left(\omega-\mathcal{J}_{0}\right)$ n'est invariant que dans une rotation d'ensemble. Dans ces conditions $1 /\left(\omega-\mathcal{H}_{0}\right)$ est un opérateur scalaire seulement vis-à-vis de $\mathbf{J}$, mais non plus vis-à-vis de $\mathbf{L}$.
$\Delta L \leqslant 2$ (cette condition est liée au rang des $Q_{i_{j}}$ ). Nous envisagerons successivement les cas $\Delta L=1,2$ et $\Delta L=0$.

2.3.1 Règles de sélection sur les transitions $\Delta L=2$ et $\Delta L=1$. - L'élément de matrice d'un opérateur scalaire $T^{0}$ est nul entre deux niveaux de $L$ différents ; donc, pour les transitions $\Delta L=1$ et $\Delta L=2, Q$ est proportionnel à un opérateur tensoriel irréductible de rang 2.

Soit $F$ le moment cinétique total (en tenant compte $\mathrm{du}$ spin électronique et éventuellement du spin nucléaire) $\left(^{3}\right)$; d'après le théorème de Wigner-Eckart, l'élément de matrice de la $q$-ième composante standard d'un opérateur tensoriel irréductible $T_{q}^{2}$ entre deux états $\mid \mathrm{g} F M_{F}>$ et $\mid \mathrm{e} F^{\prime} M_{F}^{\prime}>$ est proportionnel au coefficient de Clebsch-Gordan

$$
<F 2 M_{F} q \mid F^{\prime} M_{F}^{\prime}>\text {. }
$$

Les propriétés classiques des Clebsch-Gordan impliquent les règles de sélection suivantes :

$$
\left.\begin{array}{c}
|\Delta F| \leqslant 2 \\
M_{F}^{\prime}-M_{F}=q_{1}+q_{2} \\
F=0 \rightarrow F^{\prime}=0 \\
F=0 \rightarrow F^{\prime}=1 \\
F=\frac{1}{2} \rightarrow F^{\prime}=\frac{1}{2}
\end{array}\right\} \text { interdites. }
$$

Il y a d'autres transitions plus particularisées qui sont interdites, par exemple :

$$
\begin{aligned}
& F, M_{F}=0 \rightarrow F^{\prime}=F+1, \quad \begin{array}{l}
M_{F}^{\prime}=0 \text { interdites } \\
(F \text { entier })
\end{array} \\
& F, M_{F}=-\frac{1}{2} \rightarrow F^{\prime}=F, M_{F}^{\prime}=\frac{1}{2} \text { interdites }
\end{aligned}
$$

( $F$ demi-entier) . (15 ter)

(3) Les règles de sélection démontrées ci-dessous sont évidemment valables aussi bien pour $J$ que pour $F$. 
2.3.2 Règles de sélection sur les transitions $\Delta L=0$. - Si $L$ est nul, il faut en général conserver les deux opérateurs $T^{0}$ et $T^{2}$. Mais cette règle admet deux exceptions et ceci permet de distinguer trois cas :

a) $\mathrm{Si}$ l'on étudie des transitions telles que $M_{F}^{\prime}-M_{F} \neq 0$; alors $T^{0}$ ne peut pas coupler le niveau fondamental au niveau excité et les règles (15) et (15 ter) sont exactes.

b) $\mathrm{Si}$ le niveau fondamental et le niveau excité ont un moment cinétique orbital nul $(L=0)$. En effet, dans ce dernier cas seul l'opérateur scalaire $T^{0}$ peut coupler les deux niveaux; il s'ensuit que l'on a les règles de sélection suivantes :

$$
\begin{aligned}
\Delta F & =0 . \\
\Delta M_{F} & =0 .
\end{aligned}
$$

Il ne peut y avoir de transitions qu'entre des niveaux de même $F$ et de même $M_{F}$.

c) Dans les autres cas, c'est-à-dire $L \neq 0$ et $M_{F}^{\prime}-M_{F}=0$, les règles de sélection sont :

$$
F=0 \rightarrow \begin{gathered}
|\Delta F| \leqslant 2 \\
F^{\prime}=1 \text { interdites . }
\end{gathered}
$$

En effet ni $T^{0}$, ni $T^{2}$ ne peuvent coupler $F=0$ à $F^{\prime}=1$.

3. Applications. - Les effets que nous avons décrits ci-dessus trouveront des applications immédiates dans des études spectroscopiques de haute résolution. On peut en effet observer des raies très fines ayant la largeur naturelle; et nous avons montré d'autre part que le signal attendu doit être assez important avec une intensité du faisceau Laser tout à fait raisonnable. Pour que ces mesures aient un sens, il importe que les déplacements de fréquence provoqués par l'irradiation lumineuse, ou light-shifts [10], restent négligeables; et c'est pourquoi nous les calculons dans l'Appendice II : dans le cas des transitions à deux photons tant que l'intensité du faisceau Laser n'est pas trop forte et respecte la condition de validité de tous nos calculs (probabilité de transition $\Gamma_{\mathrm{g}}(0) \ll \Gamma_{\mathrm{e}}$ largeur naturelle), on montre que le light-shift du niveau fondamental est très petit devant la largeur naturelle $\Gamma_{\mathrm{e}}$. Le light-shift du niveau excité sera également négligeable sauf s'il existe une absorption quasi résonnante à un photon à partir du niveau excité e. Encore faut-il remarquer que si cette résonance conduit à un état du continuum éloigné du seuil d'ionisation, le light-shift sera encore généralement petit [14], c'est seulement dans le cas où l'absorption à un photon à partir du niveau excité conduit vers des niveaux (discrets ou continus) voisins du seuil d'ionisation qu'une étude approfondie s'impose.

Par rapport aux autres méthodes de spectroscopie sans largeur Doppler [6], [7], [8], la méthode proposée présente l'inconvénient d'exiger des puissances lumi- neuses nettement supérieures, mais elle ouvre de nouvelles possibilités $\left({ }^{4}\right)$ :

a) L'énergie acquise par l'atome étant la somme des énergies de plusieurs photons optiques, l'état final sera très souvent un état d'énergie élevé. La méthode peut se révéler particulièrement intéressante pour l'étude de niveaux proches du seuil d'ionisation, dont les intervalles sont inférieurs à la largeur Doppler et que l'on pourra ainsi séparer.

b) Les transitions à deux photons permettent d'atteindre certains niveaux métastables de même parité que le niveau fondamental. C'est le cas qui est représenté sur la figure $4:$ il n'existe entre les deux niveaux $\mathrm{g}$ et $\mathrm{e}$ aucun autre niveau de parité opposée ; il n'y a donc pas de transition radiative possible entre $\mathrm{e}$ et $\mathrm{g}$ et le niveau e est métastable. Mais il pourra être atteint en utilisant comme relais les niveaux d'énergie supérieure à e et de parité opposée.

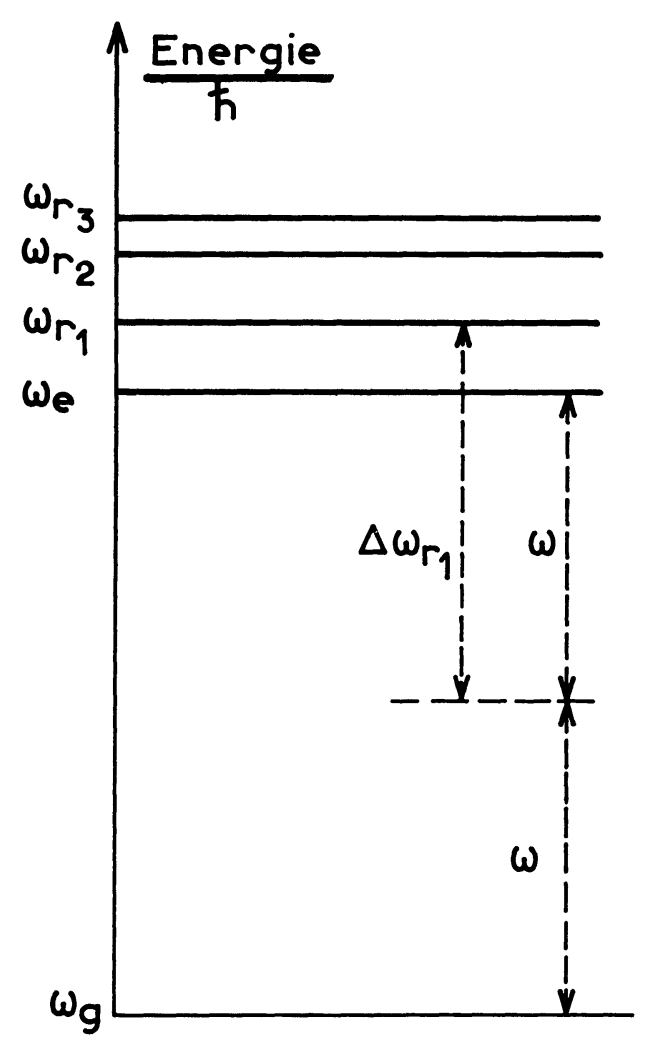

FIG. 4.

Ainsi, l'étude de la transition vers le niveau $2 \mathrm{~S}$ de l'atome d'hydrogène pourrait se révéler extrêmement intéressante lorsqu'on aura mis au point des

(4) La méthode proposée présente aussi des avantages techniques, qui devraient conduire à un meilleur rapport Signal/ Bruit que la méthode d'absorption saturée :

1) La totalité des atomes est concernée par l'expérience et non pas seulement la faible fraction d'entre eux dont la vitesse est voisine de zéro.

2) On détecte les photons réémis spontanément à partir du niveau e sur une longueur d'onde $\lambda_{\text {re }}$ différente de la longueur d'onde $\lambda$ du Laser; le signal apparaît donc sur fond noir. 
sources accordables cohérentes dans l'ultraviolet $(\lambda \simeq 2430 \AA):$ en effet, la mesure de la transition $1 \mathrm{~S} \rightarrow 2 \mathrm{~S}$ permettrait sans doute d'améliorer de façon notable la précision avec laquelle est connue la constante de Rydberg.

La formule simplifiée (11) donnant la probabilité de transition $\Gamma_{\mathrm{g}}(0)$ n'est pas valable dans le cas d'un état excité métastable, parce qu'il existe plusieurs niveaux relais correspondant à des écarts énergétiques du même ordre de grandeur $\Delta \omega_{\mathrm{r}} \gtrsim \omega$, et il faut sommer effectivement les contributions de ces différents niveaux. La formule (11) reste cependant utilisable pour une évaluation grossière de la probabilité de transition; appliquée à la transition $1 \mathrm{~S} \rightarrow 2 \mathrm{~S}$ de l'hydrogène, elle redonne bien l'ordre de grandeur obtenu à partir du calcul exact de Gontier et Trahin [15].

On voit sur la formule (11) que l'importance de l'écart énergétique $\Delta \omega_{\mathrm{r}}$ des niveaux relais est très défavorable à la production des états métastables. En revanche leur faible largeur naturelle $\Gamma_{\mathrm{e}}$ favorise leur production ; mais on ne pourra bénéficier de ce facteur favorable que dans la mesure où la pureté spectrale et la stabilité du Laser utilisé le permettront. Au total, on peut espérer que ces deux facteurs, favorable et défavorable, se compensent, et obtenir une probabilité de transition notable pour une intensité de $1 \mathrm{~W} / \mathrm{mm}^{2}$.

Nous avons montré que les transitions à deux photons ne nécessitaient pas une puissance considérable du Laser, il est possible de faire un calcul analogue dans le cas des transitions à trois photons (cf. Appendice II). Avec des valeurs raisonnables pour les forces d'oscillateurs et les écarts d'énergie, on trouve une probabilité d'environ 1 transition par seconde avec des puissances de Laser de l'ordre d'une dizaine de $\mathrm{kW}$. On peut encore remarquer que, même avec ces puissances, les déplacements de niveaux seront souvent assez petits devant la largeur du niveau excité. Ces remarques démontrent que des expériences de spectroscopie par absorption de trois photons ne sont pas utopiques.

En ce qui concerne les processus à trois photons, remarquons-le, il n'est pas nécessaire que les trois photons soient absorbés pour éliminer l'effet Doppler : on peut, en effet, imaginer une expérience où deux photons de vecteur d'onde $\mathbf{k}_{1}$ et $\mathbf{k}_{2}$ seraient absorbés et un photon de vecteur d'onde $\mathbf{k}_{\mathbf{3}}$ serait émis, cette émission pouvant être stimulée ou spontanée. Si les deux conditions suivantes, qui correspondent à la conservation de l'énergie et de l'impulsion, sont réalisées :

$$
\begin{gathered}
\hbar \omega_{\mathrm{e}}-\hbar \omega_{\mathrm{g}}=\hbar c k_{1}+\hbar c k_{2}-\hbar c k_{3} \quad \text { (cf. Fig. 5a) } \\
\hbar c \mathbf{k}_{1}+\hbar c \mathbf{k}_{2}=\hbar c \mathbf{k}_{3} \quad \text { (cf. Fig. 5b) }
\end{gathered}
$$

l'effet Doppler est éliminé.

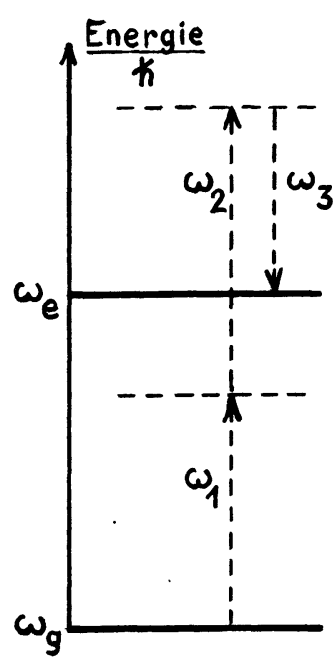

(a)

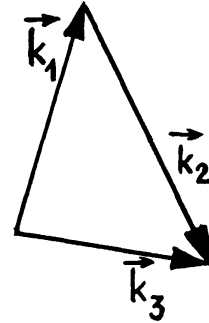

(b)
Fig. 5.

En ce qui concerne le processus où le photon $k_{3}$ est émis spontanément la probabilité d'émission pourra être assez importante si par absorption des photons $k_{1}$ et $k_{2}$ on atteint un niveau résonnant : d'autre part, on remarque que l'effet Doppler n'est éliminé que dans une direction particulière; si l'on s'écarte de cette direction, l'influence de la vitesse des atomes sur la forme de la raie d'émission deviendra de plus en plus importante.

Une autre application possible de ces transitions à plusieurs photons est la généralisation des expériences de double résonance [16] à des niveaux qui ne peuvent pas être atteints par l'absorption d'une raie de résonance. En effet, la méthode que nous proposons permet de peupler sélectivement certains sous-niveaux Zeeman de l'état excité non seulement par le jeu des polarisations comme dans les expériences de double résonance classiques, mais également du fait que dans un champ magnétique d'importance moyenne un seul sous-niveau Zeeman peut être atteint par absorption de plusieurs photons.

\section{APPENDICE I}

1. Calcul exact de l'effet Doppler résiduel dans le cas de transitions à deux photons. - Dans nos estimations de l'effet Doppler nous n'avons tenu compte que des termes du premier ordre en $v / c$; mais la théorie relativiste implique des termes du second ordre en $\left(v^{2} / c^{2}\right)$ dont nous recherchons maintenant les effets lorsque l'atome absorbe deux photons de même énergie et de sens de propagation opposés. Nous raisonnerons en termes de conservation de l'impulsion.

Plaçons-nous d'abord dans le repère du laboratoire : l'impulsion de l'atome est conservée puisque l'impulsion globale des deux photons absorbés est nulle. 
Mais l'atome excité est légèrement plus lourd que l'atome à l'état fondamental ; et à impulsion constante, sa vitesse est légèrement inférieure. C'est-à-dire qu'une faible part de l'énergie cinétique de l'atome s'ajoute à l'énergie des photons pour permettre la transition, modifiant ainsi légèrement la condition de résonance (4).

Le calcul est plus simple en passant dans le repère lié à l'atome. Nous appliquons la transformation de Lorentz au quadrivecteur impulsion-énergie de l'ensemble des deux photons (en appelant $z$ la direction de la vitesse $\mathbf{v}$ de l'atome) :

dans le repère du laboratoire

$$
\left\{\begin{array}{l}
\mathbf{p}=0 \\
E=2 \hbar \omega
\end{array}\right.
$$

dans le repère de l'atome

$$
\left\{\begin{array}{l}
p_{x}^{\prime}=p_{y}^{\prime}=0 \\
p_{z}^{\prime}=-\frac{v}{c^{2}} \frac{2 \hbar \omega}{\sqrt{1-\frac{v^{2}}{c^{2}}}} \\
E^{\prime}=\frac{2 \hbar \omega}{\sqrt{1-\frac{v^{2}}{c^{2}}}} .
\end{array}\right.
$$

L'impulsion des deux photons n'est plus exactement compensée dans le référentiel de l'atome. Lorsque l'atome absorbe les deux photons, le quadrivecteur impulsion-énergie du système global " atome + photons » étant conservé, il en résulte que l'atome acquiert une vitesse finie $\delta v$ dans le référentie où il était intialement immobile. $\left(\delta v=p_{z}^{\prime} / M\right.$ a le signe opposé à $v$; ceci correspond bien à un ralentissement de l'atome dans le repère du laboratoire.) Mais l'effet le plus important réside dans la modification de l'énergie de $E$ à $E^{\prime}$.

Ecrivons la conservation de l'énergie dans la transition :

$$
\begin{aligned}
\hbar \omega_{\mathrm{ge}}=\hbar \omega_{\mathrm{e}}-\hbar \omega_{\mathrm{g}} & \simeq E^{\prime}-\frac{1}{2} M(\delta v)^{2} \\
& \simeq \frac{2 \hbar \omega}{\sqrt{1-\frac{v^{2}}{c^{2}}}\left[1-\frac{v^{2}}{c^{2}} \frac{\hbar \omega}{M c^{2}}\right]}
\end{aligned}
$$

Le second terme du crochet, calculé à partir de $(\delta v)^{2}$, représente un faible résidu de l'effet de recul ; compte tenu du rapport $\hbar \omega / M c^{2}<10^{-9}$, il est totalement négligeable. Et la formule peut se réduire à :

$$
2 \omega \simeq \omega_{\mathrm{ge}} \sqrt{1-\frac{v^{2}}{c^{2}}} \simeq \omega_{\mathrm{ge}}\left(1-\frac{v^{2}}{2 c^{2}}\right)
$$

Le terme correctif en $v^{2} / 2 c^{2}$ vaut environ $10^{-11}$ pour les éléments légers à température ambiante ; ce rapport diminue lorsque la masse de l'élément augmente. Il est donc justifié de négliger, dans l'immense majorité des cas, cet effet Doppler du second ordre. Cependant, si l'on étudie une transition entre un niveau fondamental et un niveau métastable ce terme peut modifier la forme de la raie d'absorption : compte tenu de la distribution des vitesses, il conduit en effet à une raie décentrée et asymétrique.

\section{APPENDICE II}

1. Calcul de l'interaction d'un atome avec deux ondes monochromatiques se propageant en sens inverse. Les calculs que nous développons ci-dessous sont inspirés de la théorie de l'atome habillé [11] et plus particulièrement de l'application de cette théorie au pompage optique qui est exposé dans la thèse de J. Dupont-Roc [17]. Beaucoup des résultats que nous retrouvons ainsi sont tout à fait classiques [2], [9], [10]; mais ceci nous permet de les préciser dans notre cas particulier et de justifier les hypothèses qui ont été énumérées dans le paragraphe 2.1 .

1.1 RAPPELS SUR L'ATOME HABILlÉ. - Nous appelons $\mathcal{H}_{0}^{\prime}$ le hamiltonien du rayonnement divisé par $\hbar$. Ses états propres sont les états $\left|n_{1} n_{2}\right\rangle$ où $n_{1}$ et $n_{2}$ désignent les nombres de photons appartenant aux modes 1 et 2 :

$$
\mathcal{H}_{0}^{\prime}\left|n_{1} n_{2}\right\rangle=\left(n_{1} \omega_{1}+n_{2} \omega_{2}\right)\left|n_{1} n_{2}\right\rangle
$$

$\mathcal{H}_{0}^{\prime}$ ne tient compte que des modes « remplis» du rayonnement.

Pour tenir compte des modes vides du champ électromagnétique, qui sont responsables de la durée de vie finie des niveaux excités et que nous n'avons pas conservé dans le hamiltonien $\mathcal{J}_{0}^{\prime}$, nous ajoutons à l'énergie $\hbar \omega_{\mathrm{r}}$ d'un niveau excité une partie imaginaire $\mathrm{i} \hbar \Gamma_{\mathrm{r}} / 2 . \Gamma_{\mathrm{r}}$ est l'inverse de la durée de vie naturelle du niveau r. Nous appelons $\mathscr{H}_{0}$ le hamiltonien de l'atome libre divisé par $\hbar$; son action sur les états $|\mathrm{g}\rangle,|\mathrm{r}\rangle$ et $|\mathrm{e}\rangle$ s'écrit :

$$
\begin{aligned}
& \mathscr{H}_{\mathrm{o}}\left|\mathrm{g}>=\omega_{\mathrm{g}}\right| \mathrm{g}> \\
& \mathscr{H}_{\mathrm{o}}\left|\mathrm{r}>=\left(\omega_{\mathrm{r}}+\mathrm{i} \frac{\Gamma_{\mathrm{r}}}{2}\right)\right| \mathrm{r}> \\
& \mathscr{H}_{0}\left|\mathrm{e}>=\left(\omega_{\mathrm{e}}+\mathrm{i} \frac{\Gamma_{\mathrm{e}}}{2}\right)\right| \mathrm{e}>
\end{aligned}
$$


les états propres de $\mathscr{H}_{0}+\mathscr{H}_{0}^{\prime}$ sont les états $|\mathrm{r}\rangle\left|n_{1} n_{2}\right\rangle$ que nous noterons plus simplement $\left|\mathrm{r}, n_{1}, n_{2}\right\rangle$.

$$
\begin{aligned}
& \left(\mathcal{H}_{0}+\mathcal{H}_{0}^{\prime}\right) \mid \mathrm{r}, n_{1}, n_{2}>= \\
& \quad=\left(\omega_{\mathrm{r}}+\mathrm{i} \frac{\Gamma_{\mathrm{r}}}{2}+n_{1} \omega_{1}+n_{2} \omega_{2}\right) \mid \mathrm{r}, n_{1}, n_{2}>.
\end{aligned}
$$

En fait, le hamiltonien $\mathrm{du}$ système total " atome + photons optiques" est la somme de $\left(\mathscr{H}_{0}+\mathscr{H}_{0}^{\prime}\right)$ et d'un hamiltonien d'interaction entre l'atome et le champ; ce dernier est, lui-même la somme de deux termes $\mathscr{H}_{1}$ et $\mathscr{H}_{2}: \mathscr{H}_{1}\left(\right.$ ou $\left.\mathfrak{H}_{2}\right)$ est un opérateur qui couple les niveaux $g$ et $\mathrm{e}$ à des niveaux $\mathrm{r}$ de parité différente avec absorption ou émission d'un photon du mode 1 (ou du mode 2). C'est parce que $\mathscr{H}_{1}+\mathscr{H}_{2}$ peut au second ordre mélanger $\mid \mathrm{g}, n_{1}, n_{2}>$ avec, par exemple, $\mid \mathrm{e}, n_{1}-1, n_{2}-1>$ que le niveau fondamental acquiert une durée de vie finie. C'est le calcul de cette durée de vie du niveau fondamental perturbé par le champ électromagnétique que nous présentons maintenant.
1.2 DURÉE DE VIE DU NIVEAU FONDAMENTAL PERTURBÉ PAR LE CHAMP ÉLECTROMAGNÉTIQUE. - Calculons les états propres de $\mathcal{H}$, dans les conditions définies précédemment, en employant la théorie des perturbations appliquée à un niveau non dégénéré $\left(H_{0}+\mathcal{H}_{0}^{\prime}\right.$ étant le hamiltonien principal, $\mathcal{H}_{1}+\mathfrak{H}_{2}$ la perturbation). Il peut sembler paradoxal de considérer $\left|\mathrm{g}, n_{1}, n_{2}\right\rangle$ et $\mid \mathrm{e}, n_{1}-1, n_{2}-1>$ comme étant non dégénérés alors que ces niveaux ont mêmes énergies réelles lorsque $\delta \omega=0$. En fait, les énergies complexes de ces deux états sont différentes parce que l'un contient une partie imaginaire $\mathrm{i} \Gamma_{\mathrm{e}} / 2$ et l'autre pas ; comme cela a été remarqué par W. E. Lamb [18], tant que le couplage entre deux niveaux de même énergie (réelle) est inférieur à la différence de leurs largeurs naturelles, les fonctions d'onde des niveaux perturbés ne sont pas complètement mélangées, et il est justifié de leur appliquer la théorie des perturbations de la même façon qu'à un niveau non dégénéré. L'état propre $\left|\overline{\mathrm{g}, n_{1}, n_{2}}\right\rangle$ de $\mathfrak{H}$ a pour expression en se limitant au deuxième ordre de la série de perturbations :

$$
\begin{aligned}
&\left|\overline{\mathrm{g}, n_{1}, n_{2}}>=\right| \mathrm{g}, n_{1}, n_{2}>+\sum_{\mathrm{r}}\left[\alpha_{1}(\mathrm{r})\left|\mathrm{r}, n_{1}-1, n_{2}>+\alpha_{2}(\mathrm{r})\right| \mathrm{r}, n_{1}, n_{2}-1>\right]+ \\
& \quad+\frac{R_{12}}{2 \omega-\omega_{\mathrm{ge}}-\mathrm{i} \frac{\Gamma_{\mathrm{e}}}{2}}\left|\mathrm{e}, n_{1}-1, n_{2}-1>+\frac{R_{11}}{2 \omega-2 k v_{x}-\omega_{\mathrm{ge}}-\mathrm{i} \frac{\Gamma_{\mathrm{e}}}{2}}\right| \mathrm{e}, n_{1}-2, n_{2}> \\
&+\frac{R_{22}}{2 \omega+2 k v_{x}-\omega_{\mathrm{ge}}-\mathrm{i} \frac{\Gamma_{\mathrm{e}}}{2}}\left|\mathrm{e}, n_{1}, n_{2}-2>+\frac{P_{12}}{2 k v_{x}}\right| \mathrm{g}, n_{1}+1, n_{2}-1> \\
&+\frac{P_{21}}{\left(-2 k v_{x}\right)} \mid \mathrm{g}, n_{1}-1, n_{2}+1>+\cdots
\end{aligned}
$$

Les divers symboles, $\alpha_{1}(\mathrm{r}), R_{12}$, etc..., apparaissant dans cette formule sont tout à fait classiques, et sont détaillés dans le tableau I. Dans l'ensemble des termes du second ordre nous avons négligé ceux qui ne sont pas résonnants ou quasi résonnants ; et c'est la raison pour laquelle, compte tenu de l'hypothèse (2), n'apparaît que le seul état e pour lequel $\delta \omega=\omega-\frac{\omega_{\mathrm{ge}}}{2}$ est voisin de zéro.

Le champ électromagnétique mélange la fonction d'onde du niveau $\left|\mathrm{g}, n_{1}, n_{2}\right\rangle$ à celles de divers autres états : le couplage avec les niveaux

$$
\left|\mathrm{r}, n_{1}-1, n_{2}\right\rangle \quad \text { et } \quad\left|\mathrm{r}, n_{1}, n_{2}-1\right\rangle
$$

est responsable du light-shift [10]. Le couplage avec les niveaux $\left|\mathrm{e}, n_{1}-2, n_{2}\right\rangle, \mid \mathrm{e}, n_{1}-1, n_{2}-1>$, | e, $n_{1}, n_{2}-2>$ introduit une durée de vie finie du niveau fondamental; en effet la probabilité, le système étant dans l'état $\left|\overline{\mathrm{g}, n_{1}, n_{2}}\right\rangle$, de se trouver dans l'état atomique e est égale à :

$$
p=\left|\frac{R_{12}}{2 \delta \omega-\mathrm{i} \frac{\Gamma_{\mathrm{e}}}{2}}\right|^{2}+\left|\frac{R_{11}}{2 \delta \omega-2 k v_{x}-\mathrm{i} \frac{\Gamma_{\mathrm{e}}}{2}}\right|^{2}+\left|\frac{R_{22}}{2 \delta \omega+2 k v_{x}-\mathrm{i} \frac{\Gamma_{\mathrm{e}}}{2}}\right|^{2} .
$$

La probabilité pour un système se trouvant dans l'état de e disparaître est égale à $\Gamma_{\mathrm{e}}$ pour une unité de temps; donc $\mid \overline{\mathrm{g}, n_{1}, n_{2}}>$ acquiert une durée de vie $1 / \Gamma_{\mathrm{g}}$ avec :

$$
\Gamma_{\mathrm{g}}=\Gamma_{\mathrm{e}}\left[\frac{\left|R_{12}\right|^{2}}{4 \delta \omega^{2}+\frac{\Gamma_{\mathrm{e}}^{2}}{4}}+\frac{\left|R_{11}\right|^{2}}{\left(2 \delta \omega-2 k v_{x}\right)^{2}+\frac{\Gamma_{\mathrm{e}}^{2}}{4}}+\frac{\left|R_{22}\right|^{2}}{\left(2 \delta \omega+2 k v_{x}\right)^{2}+\frac{\Gamma_{\mathrm{e}}^{2}}{4}}\right] .
$$




\section{TABLEAU I}

Coefficients apparaissant dans les formules (A.4) et (A.5) ; on a remplacé les dénominateurs d'énergie $\omega \pm k v_{z}-\omega_{\mathrm{gr}}-\mathrm{i} \Gamma_{\mathrm{r}} / 2$ par $\omega-\omega_{\mathrm{gr}}$, ce qui est justifié par l'hypothèse (3).

$$
\begin{aligned}
& \alpha_{1}(r)=\frac{\left.\varangle, n_{1}-1, n_{2}\left|F_{1}\right| g, n_{1}, n_{2}\right\rangle}{\omega-\omega_{g r}} \\
& \alpha_{2}(r)=\frac{\left\langle r, n_{1}, n_{2}-1\left|\pi_{2}\right| g, n_{1}, n_{2}\right\rangle}{\omega-\omega_{g r}} \\
& R_{12}=\sum_{r} \frac{\left\langle e, n_{1}-1, n_{2}-1\left|H_{1}\right| r, n_{1}, n_{2}-1\right\rangle\left\langle r, n_{1}, n_{2}-1\left|r_{2}\right| g, n_{1}, n_{2}\right\rangle+\left\langle e, n_{1}-1, n_{2}-1\left|H_{2}\right| r, n_{1}-1, n_{2}><r, n_{1}-1, n_{2}\left|H_{1}\right| g, n_{1}, n_{2}\right\rangle}{\omega-\omega_{g r}} \\
& R_{11}=\sum_{r} \frac{\left\langle e, n_{1}-2, n_{2}\left|\pi c_{1}\right| r, n_{1}-1, n_{2}><r, n_{1}-1, n_{2}\left|H_{1}\right| g, n_{1}, n_{2}\right\rangle}{\omega-\omega_{g r}} \\
& R_{22}=\sum_{r} \frac{\left\langle e, n_{1}, n_{2}-2\left|\pi_{2}\right| r, n_{1}, n_{2}-1><r, n_{1}, n_{2}-1\left|\pi_{2}\right| g, n_{1}, n_{2}\right\rangle}{\omega-\omega_{g r}} \\
& P_{12}=\sum_{r} \frac{\left\langle g, n_{1}+1, n_{2}-1\left|\tilde{x}_{1}\right| r, n_{1}, n_{2}-1\right\rangle\left\langle r, n_{1}, n_{2}-1\left|x_{2}\right| g, n_{1}, n_{2}\right\rangle}{\omega-\omega_{g r}} \\
& P_{21}=\sum_{r} \frac{\left\langle g, n_{1}-1, n_{2}+1\left|F_{2}\right| r, n_{1}-1, n_{2}<<r, n_{1}-1, n_{2}\left|F_{1}\right| g, n_{1}, n_{2}\right\rangle}{\omega-\omega_{g r}}
\end{aligned}
$$

Remarquons que cette formule, tout comme le développement (A.4) n'est valable que si les divers $\left|R_{i j}\right|(i, j=1$ ou 2$)$ sont très petits devant la largeur $\Gamma_{\mathrm{e}}$ du niveau excité. On retrouve ainsi la condition de Lamb et notre hypothèse (4) :

$$
\left|R_{i j}\right| \ll \Gamma_{\mathrm{e}} \rightarrow \Gamma_{\mathrm{g}} \ll \Gamma_{\mathrm{e}}
$$

On peut noter également que le champ électromagnétique couple $\mid \mathrm{g}, n_{1}, n_{2}>$ à $\mid \mathrm{g}, n_{1}-1, n_{2}+1>$; ceci correspond à un processus Raman stimulé où l'atome absorbe un photon se propageant dans un sens et émet un photon se propageant en sens inverse. Puisque la durée de vie de ces deux niveaux est identique, le couplage mélange complètement les niveaux lorsqu'ils ont même énergie, c'est-à-dire quand $v_{x}$ est voisin de zéro. On vérifie aisément que le développement (A.4) n'est pas valable dans ce cas. C'est ce qui nécessite l'hypothèse (5).

On retrouve l'expression (A.5) d'une autre manière dans le paragraphe suivant, en même temps que l'on calcule les déplacements de niveaux d'énergie provoqués par la lumière ou light-shifts.

1.3 Light-ShifT. - Pour connaître les déplacements de niveaux, nous calculons l'énergie du niveau perturbé $\left|\overline{\mathrm{g}, n_{1}, n_{2}}\right\rangle$. Le résultat présenté ci-dessous provient d'un développement au $4^{\mathrm{e}}$ ordre ; en fait le calcul est relativement simplifié par des considérations évidentes de parité, montrant que seuls les termes d'ordre pairs sont non nuls. Soit $\hbar \bar{\omega}_{\mathrm{g}}$ l'énergie du niveau perturbé :

$\bar{\omega}_{\mathrm{g}}=\omega_{\mathrm{g}}+n_{1} \omega_{1}+n_{2} \omega_{2}+\Delta E(2)-\mathrm{i} \frac{\Gamma(4)}{2}+\Delta E(4)\left(^{5}\right)$

$\Delta E(2)$ est obtenu au second ordre de perturbation. C'est le déplacement de niveau calculé habituellement [10] :

$$
\begin{gathered}
<\mathrm{g}, n_{1}, n_{2}\left|\mathcal{H}_{1}\right| \mathrm{r}, n_{1}-1, n_{2}><\mathrm{r}, n_{1}-1, n_{2}\left|\mathcal{H}_{1}\right| \mathrm{g}, n_{1}, n_{2}>+ \\
\Delta E(2)=\sum_{\mathrm{r}} \frac{+<\mathrm{g}, n_{1}, n_{2}\left|\mathcal{H}_{2}\right| \mathrm{r}, n_{1}, n_{2}-1><\mathrm{r}, n_{1}, n_{2}-1\left|\mathcal{H}_{2}\right| \mathrm{g}, n_{1}, n_{2}>}{\omega-\omega_{\mathrm{gr}}}
\end{gathered}
$$

Ce déplacement $\Delta E(2)$ est donné par une expression analogue à celle des coefficients $R_{i j}$ (cf. Tableau I); et il sera généralement du même ordre de grandeur. Compte tenu de la condition (A.6), on voit que dans le cadre de nos approximations ce déplacement reste
(5) Le terme imaginaire du second ordre $\mathrm{i} \Gamma(2) / 2$ est nul parce que nous avons négligé la partie imaginaire au dénominateur des coefficients $\alpha_{1}(\mathrm{r})$ et $\alpha_{2}(\mathrm{r})$ (cf. Tableau I). Si nous tenions compte de cette partie imaginaire, nous obtiendrions un terme $\Gamma(2)$ différent de zéro, exprimant l'effet Raman spontané. 
très petit devant la largeur naturelle du niveau excité :

$$
|\Delta E(2)| \sim\left|R_{i j}\right| \rightarrow|\Delta E(2)| \ll \Gamma_{\mathrm{e}} .
$$

- $\mathrm{i} \Gamma(4) / 2$ est la partie imaginaire de l'énergie du niveau $\left|\overline{\mathrm{g} n_{1} n_{2}}\right\rangle$, elle correspond à une durée de vie finie pour le niveau $\left|\overline{\mathrm{g} n_{1} n_{2}}\right\rangle$; on trouve évidemment que $\Gamma(4)$ est identique à la largeur $\Gamma_{\mathrm{g}} \mathrm{du}$ niveau $\mid \overline{\mathrm{g} n_{1}} n_{2}>$ calculée au paragraphe précédent (formule (A.5)).

- Le déplacement de niveau d'ordre 4 est essentiellement la somme de termes provenant du couplage de $\left|\mathrm{g} n_{1} n_{2}\right\rangle$ avec des niveaux quasi résonnants.

$$
\Delta E(4)=\frac{2 \delta \omega\left|R_{12}\right|^{2}}{4 \delta \omega^{2}+\frac{\Gamma_{\mathrm{e}}^{2}}{4}}+\frac{\left(2 \delta \omega-2 k v_{x}\right)\left|R_{11}\right|^{2}}{\left(2 \delta \omega-2 k v_{x}\right)^{2}+\frac{\Gamma_{\mathrm{e}}^{2}}{4}}+\frac{\left(2 \delta \omega+2 k v_{x}\right)\left|R_{22}\right|^{2}}{\left(2 \delta \omega+2 k v_{x}\right)^{2}+\frac{\Gamma_{\mathrm{e}}^{2}}{4}}+\frac{\left|P_{12}\right|^{2}-\left|P_{21}\right|^{2}}{2 k v_{x}} .
$$

Les coefficients $P_{12}$ et $P_{21}$ sont pratiquement toujours égaux, ce qui assure la nullité du dernier terme. Le premier terme change de signe avec $\delta \omega$ et ne contribue donc pas à déplacer la raie fine. Les deuxième et troisième termes sont de signes opposés, pour $\delta \omega$ voisin de zéro, et ils s'annuleront l'un l'autre dans un certain nombre de cas; de toute façon, il est facile de montrer que $|\Delta E(4)| \ll|\Delta E(2)|$ en tenant compte de la condition (A.6) et du fait que $|\Delta E(2)| \sim\left|R_{i j}\right|$. On en conclut que généralement le light-shift du niveau fondamental est négligeable.

Le calcul du déplacement de l'état excité se fait de façon tout à fait analogue; on montre que les termes d'ordre 4 sont petits devant $\Gamma_{\mathrm{e}}$. La partie du déplacement au $2^{\mathrm{e}}$ ordre liée à l'émission puis à l'absorption d'un photon est également, dans la grande majorité des cas, petite devant $\Gamma_{\mathrm{e}}$. Il est, en revanche, plus difficile d'évaluer le terme lié à l'absorption puis à l'émission d'un photon; en effet, les dénominateurs d'énergie qui interviennent dans cette composante du light-shift sont $\left(\omega+\omega_{\mathrm{e}}-\omega_{\mathrm{r}}\right)$ : ils sont différents des $\left(\omega-\omega_{\mathrm{gr}}\right)$. Il faudra donc faire une évaluation précise du déplacement lorsqu'il existe des niveaux $r$ plus excités que e et qui sont quasi-résonnants $\left(\omega_{\mathrm{r}} \simeq \omega_{\mathrm{e}}+\omega\right)$.

1.4 Calcul de la probabilité De transition a RÉSONANCE. - Nous nous intéressons seulement aux transitions sans effet Doppler. A la résonance exacte $(\delta \omega=0)$ la probabilité prend la valeur :

$$
\Gamma_{\mathrm{g}}(0)=\frac{4}{\Gamma_{\mathrm{e}}}\left|R_{12}\right|^{2} .
$$

Le champ électromagnétique est traditionnellement quantifié dans la jauge où le potentiel scalaire est nul, et le hamiltonien d'interaction entre l'atome et le rayonnement s'écrit alors - $(q / m)(\mathbf{p . A})$. Mais on peut montrer son équivalence avec le hamiltonien exprimé à l'aide du moment dipolaire électrique $\mathbf{D}=\sum q \mathbf{r}$ (cf. en particulier Bunkin [13]).

Si les champs électriques des deux ondes, ont les vecteurs unitaires $\varepsilon_{1}$ et $\varepsilon_{2}$, nous obtenons :

$$
R_{12}=\frac{\omega \sqrt{n_{1} n_{2}}}{2 \varepsilon_{0} \hbar V}\left[\sum_{\mathrm{r}} \frac{\left.\left\langle\mathrm{e}\left|\mathbf{D} . \varepsilon_{1}\right| \mathrm{r}\right\rangle\left\langle\mathrm{r}\left|\mathbf{D} . \varepsilon_{2}\right| \mathrm{g}\right\rangle+<\mathrm{e}\left|\mathbf{D} . \varepsilon_{2}\right| \mathrm{r}\right\rangle\left\langle\mathrm{r}\left|\mathbf{D} . \varepsilon_{1}\right| \mathrm{g}\right\rangle}{\omega-\omega_{\mathrm{gr}}}\right] .
$$

1.5 GÉNÉRALISATION AUX TRANSITIONS A 3 PHOTONS. - Les calculs précédents se généralisent aisément aux transitions à un nombre quelconque de quanta; par exemple, dans le cas d'une transition à trois quanta on trouve qu'à résonance la probabilité pour l'atome d'absorber trois photons de pulsations $\omega_{1}$, $\omega_{2}$ et $\omega_{3}$ et de polarisations $\boldsymbol{\varepsilon}_{1}, \boldsymbol{\varepsilon}_{2}$ et $\boldsymbol{\varepsilon}_{3}$ est égale à :

$$
\Gamma_{\mathrm{g}}(0)=\frac{4}{\Gamma_{\mathrm{e}}}|R|^{2}
$$

$R$ est le couplage entre le niveau fondamental et le niveau excité :

$$
R=\frac{\sqrt{n_{1} \omega_{1} n_{2} \omega_{2} n_{3} \omega_{3}}}{\left(2 \varepsilon_{0} \hbar V\right)^{3 / 2}}\left[\sum_{\substack{r, \mathrm{r}^{\prime} \\\{, j, k\}}} \frac{\left\langle\mathrm{e}\left|\mathbf{D} . \varepsilon_{i}\right| r><\mathrm{r}\left|\mathbf{D} . \varepsilon_{j}\right| r^{\prime}><r^{\prime}\left|\mathbf{D} . \varepsilon_{k}\right| \mathrm{g}\right\rangle}{\left(\omega_{k}-\omega_{\mathrm{gr}}\right)\left(\omega_{\mathrm{re}}-\omega_{\mathrm{i}}\right)}\right] .
$$

Dans cette somme $\{i, j, k\}$ signifie que l'on doit faire la somme sur toutes les permutations possibles des indices $\{1,2,3\}$.
Les expressions (A.12) et (A.13) sont valables si $|R|$ est petit devant la largeur du niveau excité $\Gamma_{\mathrm{e}}$. Mais, à la différence du cas à 2 photons, cette condition 
est insuffisante pour évaluer les déplacements de niveaux. Si l'on suppose que tous les écarts d'énergie sont du même ordre de grandeur:

$$
\omega_{k}-\omega_{\mathrm{gr}^{\prime}} \simeq \omega_{\mathrm{re}}-\omega_{i} \simeq \Delta \omega,
$$

si l'on suppose en outre que les trois ondes électromagnétiques ont même fréquence et même intensité, on en déduit la relation approchée, suivante entre le couplage $R$ et le light-shift, qui est toujours donné par une expression analogue à (A.8) :

$$
(\Delta E)^{3} \sim \Delta \omega|R|^{2} .
$$

On voit qu'il convient d'imposer à $|R|$ une condi- tion plus restrictive que $|R| \ll \Gamma_{\mathrm{e}}$ si l'on veut que $\Delta E$ soit petit devant $\Gamma_{\mathrm{e}}$, à savoir $|R| \ll \Gamma_{\mathrm{e}} \sqrt{\Gamma_{\mathrm{e}} / \Delta \omega}$, ce qui entraîne

$$
\Gamma_{\mathrm{g}}(0)<\Gamma_{\mathrm{e}}\left(\frac{\Gamma_{\mathrm{e}}}{\Delta \omega}\right)
$$

Les déplacements de fréquence des transitions à 3 photons ne seront négligeables que dans des conditions où le nombre de ces transitions restera très faible.

Remerciements. - Nous remercions M. CohenTannoudji pour ses précieuses suggestions au sujet de cet article.

\section{Bibliographie}

[1] Winter, J. M., Thèse, Annls de Phys. 4 (1959) 745.

Kusch, P., Phys. Rev. 93 (1954) 1022 ; 101 (1956) 627.

[2] Bonch-Bruevich, A. M. et Khodovoi, V. A., Sov. Phys. Usp. 85 (1965) 3.

Gold, A., Two Photons Spectroscopy dans le cours de l'Ecole Enrico Fermi (Varenna, 1967).

WoRLOCK, J. M., dans Laser Handbook, (North Holland) 1972, tome II, p. 1323.

[3] Abella, I. D., Phys. Rev. Lett. 9 (1962) 453.

Delone, G. A. et Delone, N. B., JeTP Lett. 10 (1969) 265.

Braunlich, P., Hall, R. et Lambropoulos, P., Phys. Rev. A 5 (1972) 1013.

Held, B., Mainfray, G., Manus, C., Morellec, C. et SANChez, F., Phys. Rev. Lett. 30 (1973) 425.

[4] Bonch-Bruevich, A. M., Khodovoi, V. A. et Khronov, V. V., JETP Lett. 14 (1971) 333.

Agostini, P., Bensoussan, P. et Boulassier, J. C., Opt. Commun. 5 (1972) 293.

[5] Vasilenko, L. S., Chebotaev, V. P. et Shishaev, A. V. JETP Lett. 12 (1970) 161.

[6] Brewer, R. G., Kelley, M. J. et Javan, A., Phys. Rev. Lett. 23 (1969) 559.

Hansch, T. W., Levenson, M. D. et Schawlow, A. L. Phys. Rev. Lett. 26 (1971) 946.

Haroche, S. et Hartmann, F., Phys. Rev. A 6 (1972), 1299.
[7] Feld, M. S. et Javan, A., Phys. Rev. 117 (1969) 540. Feldman, B. J. et Feld, M. S., Phys. Rev. A 5 (1972) 899.

Skribanowitz, N., Kelly, M. J. et Feld, M. S., Phys. Rev. A 6 (1972) 2302.

[8] Picque, J. L. et Vialle, J. L., Opt. Commun. 5 (1972) 402.

[9] Goppert-Mayer, M., Ann. Phys. 9 (1931) 273.

[10] Cohen-Tannoudj, C., Thèse, An.lls de Phys. 7 (1962) 423-469.

Alexandrov, E. B., Bonch-Bruevitch, A. M., Kostin, N. N., Khodovol, V. A., JETP Lett. 3 (1966) 53.

Platz, P., Appl. Phys. Lett. 14 (1969) 168.

[11] Cohen-Tannoudi, C., Cargèse, Lectures on Physics (Gordon and Breach) 1967.

Haroche, S., Thèse, Annls de Phys. 6 (1971) 189-327.

[12] Shore, B. W. et Menzel, P. H., Principles of Atomic Spectra (J. Wiley and Sons) 1968, 445.

[13] Bunkin, F. V., JETP 23 (1966) 1121.

[14] Dupont-Roc, J., non publié.

[15] Gontier, Y. et Trahin, M., Phys. Rev. Lett. 36A (1971) 463.

[16] Brossel, J. et Bitter, F., Phys. Rev. 83 (1952) 210.

[17] Dupont-Roc, J., Thèse (1972).

[18] Lamb, W. E., Phys. Rev. 85 (1952) 259. 